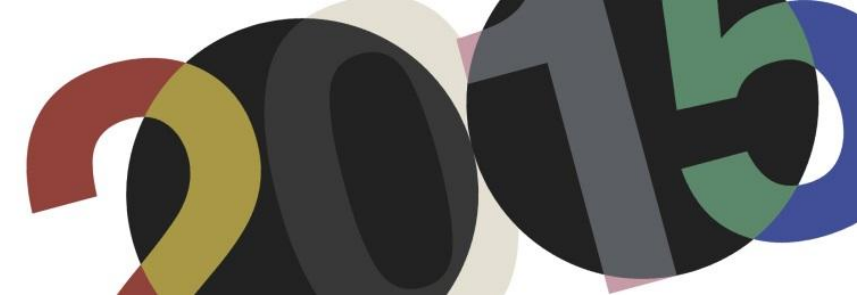

DOI: http://dx.doi.org/10.4995/LC2015.2015.728

\title{
Possibles liens avec le monde Antique. La suggestion des ruines dans les œuvres de Le Corbusier: de l'architecture Romaine au bâtiment de la Haute-Cour de Justice de Chandigarh
}

\author{
C. Roma
}

PhD candidate in Architecture - Theories and Project, Sapienza Università di Roma

\begin{abstract}
Résumé: La recherche s'intéresse à la formation de Le Corbusier et à sa capacité d'abstraction au travers des mémoires, images liées à la connaissance du monde antique; un approfondissement qui traite le lien entre les ouvres du Maître et l'étude de l'architecture romaine, soulignant ainsi deux clés de lecture: une liée à l'archétype des modèles classiques, et une seconde liée aux ruines et à son paysage archéologique. Si la première laisse apparaitre clairement la composante rationnelle, volonté de poursuivre une architecture universelle, dans laquelle s'affirme l'utilisation de la raison que seuls les archétypes peuvent enseigner; la seconde tente de délimiter la relation entre le pouvoir évocateur de la ruine et les suggestions personnelles de l'architecte. Parcourant les phases de sa formation, la recherche se concentre sur les connaissances que le jeune Jeanneret acquiert durant son séjour allemand (1910-1911), première phase d'étude de l'architecture romaine qui alimente un intérêt destiné à se développer grâce à l'expérience du Voyage d'Orient et à l'approfondissement de certaines sources, tels que les vues de Piranesi. Cet approfondissement se retrouve dans de nombreux dessins et réflexions contenus dans les carnets 4 et 5 rédigés durant le séjour romain. Ces expériences représenteront une source d'inspiration pour sa production architecturale et théorique, présuppositions qui semblent apparaitre dans certaines cuvres de l'architecte y compris la Haute Cour de Justice de Chandigarh en 1952.
\end{abstract}

\begin{abstract}
The research focuses on Le Corbusier educational process and on his ability to abstract, through his memories, the images related to the knowledge of the Ancient World; a study that addresses the clear connection between the works of Le Corbusier and his knowledge of Roman architecture, outlining two interpretations: the first one linked to the archetype of classic models, and the second one to ruins and archaeological landscape. The first interpretation clearly illustrates the rational perspective, the will to pursue an universal architecture distinguished by use of reason, that only archetypes can teach, whereas the second interpretation attempts to outline the relationship between the evocative influence of the ruin and the personal suggestions of the architect. Retracing the steps of his educational process, the research investigates the knowledge acquired by the young Jeanneret during his German stay (1910-1911). This is the first phase of the study of Roman architecture, that inspires him an interest that will be pursued later through the experience of the Voyage d 'Orient and the study of some sources, such as Piranesi's views. This learning is reflected in numerous drawings and reflections contained in Carnet 4 and in Carnet 5, elaborated during his stay in Rome. These experiences will be a source of inspiration for his architectural production and theoretical assumptions, and they seem to be reflected in some works of the architect, as the Chandigarh Haute Cour of 1952.
\end{abstract}

Mots-clés: ruines; architecture Romaine; Villa Adriana; Haute Cour.

Keywords: ruins; Roman architecture; Villa Adriana; Haute Cour.

\section{Introduction}

Cet article affronte le rapport d'intérêt et d'étude que Charles Edouard Jeanneret a tissé, dès les premières années de sa formation, avec l'architecture Romaine, dès les premières années de sa formation, qui deviendront source de suggestion et d'inspiration qu'il reproposera dans son œuvre. Sont prises en considération les sources primaires, Vers une Architecture, Le poème de l'angle droit, Euvre complète, Le Voyage d'Orient et les Carnets d'où l'on perçoit l'intérêt qu'il a eu, durant sa formation, pour les ruines de l'architectures romaines, manifesté à 
travers l'étude des textes Les Entretiens de la Villa de Rouet de Alexander Criniglia-Vaneyre, deux volumes, les œuvres de Pierre Gusman, Dictionnaire raisonné de l'architecture française du XIe au XVIe siècle de Viollet-leDuc, et le dessin de la ruine romaine et le détail du Praetorium de Villa Adriana.

Ce même sujet est recherché dans les sources secondaires, en considérant comme texte basilaire le volume L'Italia di Le Corbusier de Maria Talamona, édité en 2012, représentant une récente et exhaustive de la formation de l'architecte en Italie, tant par son approche que par sa connaissance du paysage archéologique italien. Un deuxième texte, Le Corbusier a Villa Adriana, par Eugenio Gentili Tedeschi et Giovanni Denti (sous la direction de Annalisa Mauri) représente le lien essentiel pour reconstruire la visite à la Villa de Adriano.

L'analyse des sources conduit à supposer un fructueux système de relation entre Le Corbusier et l'architecture romaine, à travers la figure de Piranesi, dont Le Corbusier découvre les vues d'abord à travers les textes, ensuite durant la visite au Unterrichtsanstalt des Königlichen Kunstgewerbemuseum et enfin à Rome. Ce rapport définit un double intérêt, renforcé dans l'admiration pour la rigueur géométrique de l'architecture classique, qui sera théorisée dans un similaire ordre géométrique Moderne, et dans la fascination pour les ruines sous la forme dans laquelle elles sont parvenues à l'époque récente. La duplicité d'intérêt pour l'architecture Antique, et en particulier pour Villa Adriana, se reflète aussi dans sa production architecturale sous forme d'inspirations diverses mais coexistantes, dans un mélange d'ordre, de pureté formelles et de rugosité épidermique, comme montré dans le projet de la Haute Cour de Chandigarh.

\section{Les acquis du Monde Antique}

"Pleine main j'ai reçu...Pleine main je donne"

L'interprétation que Le Corbusier, donnait de sa capacité à donner et recevoir du monde qui l'entourait, se concrétise dans une déclaration contenue dans le dernier chapitre du poème Le Poème de l'Angle Droit ${ }^{2}$. La main ouverte est un geste d'altruisme, un signe de paix pour l'humanité - la main "ouverte pour donner, ouverte pour recevoir",3.

Ce concept est l'une des plus importantes pensées que Le Corbusier développe durant l'âge mûr à l'occasion de la rédaction du texte cité. Ce concept est élaboré alors qu'il réalise le projet de la cité de Chandigarh. La grande sculpture, emblème proposé pour le développement de la nouvelle ville, est porteuse de nombreuses significations: elle interprète les débats politiques du pays, la difficile recherche d'indépendance que l'Inde est en train de conquérir, et la philosophie professée par Gandhi ${ }^{4}$.

Mais la main ouverte semble aussi rappeler le sens d'altruisme d'un homme qui a su recevoir d'autres cultures, et qui, grâce à sa capacité d'évolution, a su répondre à travers son engagement démontré dans les dynamiques architecturales développées au cours du XIX ${ }^{5}$.

\footnotetext{
${ }^{1}$ Le Corbusier, Le poème de l'angle droit, Paris: 1ed., Editions Verve, 1955

${ }^{2} i d$.

${ }^{3}$ id.

${ }^{4}$ Kurtis, W.JR., Il moderno e l'arcaico, o le ultime opere, p. 297 in Abram, J.; Bancon, M.; Baudouï, R., et al., Enciclopedia Le Corbusier, Milano: Electa, 1988. Argumentations sur les possibles connections entre l'emblème de la "main ouverte" et les questions liées aux évènements politiques qui secouent l'Inde après l'indépendance anglaise.

${ }^{5}$ Tentori, F., I viaggi come una mano aperta, pp.62-69 dans Gravagnuolo, B., Le Corbusier e l'Antico. Viaggi nel Mediterraneo, Napoli: Electa, 1997
} 
L'image de la Main Ouverte, est la métaphore qui montre le mieux l'aspect humain du Maître ; elle va outre les superstructures culturelles qui ont rendu l'architecte emblème incontesté de la rationalité. Il en ressort donc une sensibilité plus intime, qui embrasse son engagement pour les complexes évènements d'un pays qui se prépare à renaître.

Transposant le terme recevoir aux questions liées à la première phase d'apprentissage de l'architecture, et soulignant certains moments fondamentaux de la croissance du jeune architecte, on peut reparcourrir les premières élaborations théoriques vouées à l'identification des archétypes architecturaux ; terrain fertile sur le quel le jeune Charles-Edouard Jeanneret basera ses premières convictions qui convergeront dans les articles rédigés pour la revue L'Esprit Nouveau ${ }^{6}$ et qui mieux se concrétisent dans le texte Vers une architecture ${ }^{7}$.

\section{La rencontre avec l'Architecture Romaine}

Jeanneret est un peu plus qu'un garçon quand, sous le conseil de son maître Charles L'Eplattenier, il décide, le 12 juin 1905, d'interrompre les études commencées dans l'École d'Art à Chaux-de-Fonds, en dépit de son engagement et d'excellents résultats obtenus au cours des trois premières années de sa formation. Bien que ses études auraient dû faire de lui un graveur-ciseleur pour des caisses d'horloge, lui assurant un emploi dans l'industrie du pays, le jeune Jeanneret, abandonne l'école et poursuit un rêve plus grand, l'étude de l'architecture ${ }^{8}$.

Après ce changement, il fait ses premiers pas dans le monde de l'architecture dans son pays natale, grâce au contact d'un architecte locale, fourni par son maître; c'est alors que le jeune réalise les premiers dessins de détails constructifs, selon la méthode rationaliste décrite par Viollet-le-Duc ${ }^{9}$.

Enfin l'Eplattenier encourage le jeune Charles-Edouard Jeanneret à entreprendre le Grand Tour le 3 septembre 1907, voyage qui, pendant quatre ans, le conduira dans les principaux centres de culture européen. Les séjours commencent avec l'étude théorique de l'architecture que le jeune entreprend en Allemagne. Moments cruciaux durant lesquels il mûrit le penchant pour l'étude des architectures romaines qui semble l'éloigner du premier intérêt pour les composantes plus décoratives, héritage de la première formation suisse cultivé en occasion de son premier voyage en Toscane, en $1907^{10}$.

La relation que Jeanneret établit avec l'Italie, et en particulier avec l'architecture romaine, a été amplement explorée par Marida Talamona, en particulier lors de l'organisation de l'exposition L'Italia di Le Corbusier ${ }^{11}$. Les études et recherches dérivant de cette initiative ont fourni de soigneuses reconstructions sur les années dédiées, par Le Corbusier, à l'étude et à l'analyse des antiques architectures romaines, outils qui ont soutenu l'analyse ici proposée.

Effectuant une reconstruction des évènements qui soudent les rapports entre les architectures antiques et la production architecturale de Le Corbusier, il apparaît le caractère du paysage archéologique romain perçu non

\footnotetext{
${ }^{6}$ Revue d'art "L'Esprit Nouveau" fondée en mars 1920 à Paris par Charles Edouard Jeanneret - qui lors de cette occasion prendra le pseudonyme de Le Corbusier - avec l'artiste Amédée Ozenfant; comme outil de communication du manifeste Après le Cubisme

${ }^{7}$ Le Corbusier, Vers une architecture, Paris, 1923

${ }^{8}$ Petit, J., Le Corbusier lui-même, Paris: 1ed. qui était Le Corbusier, 1968 pp. 24-44. et Dumont, M.-J., Dall'art Nouveau all'avanguardia, tasselli di una formazione, dans L'Italia di Le Corbusier, par Talamona M., L'Italia di Le Corbusier, Milano: Mondadori Electa S.p.A, 2012, pp. 19-30

${ }^{9}$ id., p 24

${ }^{10}$ Passati, F., Toscana, p. 41-50, dans Talamona, M., 2012 op.cit.

${ }^{11}$ Exposition, L'Italia di Le Corbusier, de Marida Talamona, Roma: MAXXI Museo delle Arti del XXI secolo, 2012
} 
seulement comme une extraction des archétypes des constructions classiques. Mais apparaît aussi l'inspiration d'un paysage confus, constitué de ruines qui s'accumulent à l'intérieur des murs de l'Urbe, et qui respirent dans la campagne romaine de Villa Adriana à Tivoli.

En souhaitant effectuer une reconstruction des premiers contacts que le jeune Jeanneret a eu avec la connaissance des architectures classiques, il faut tenter de reparcourir les connaissances acquises à travers les textes consultés durant les études de jeunesse, du moins les plus significatifs, mais bien plus encore la préparation entamée en vue du voyage. Durant le printemps de 1908 son intérêt est captivée par le livre L'architecture romane de Edouard Corroyer (1888) au point de lui dédier un cahier entier, rempli de croquis et notes pour la compréhension de l'antiquité ${ }^{12}$.

Il entre en contact avec le lettré William Ritter ${ }^{13}$ - figure significative pour la maturation de tels intérêts - qui conseil au jeune l'étude de Les Entretiens de la villa de Rouet ${ }^{14}$ de Alexander Criniglia-Vaneyre, œuvre qui "marquera un tournant dans sa formation et le passage de la tradition gothique à celle classique"15.

"Notre âme est classique, en effet elle ne peut qu'évoluer vers une formule gréco-latine (...) j'avais étouffé tout ce qui pouvait m'éloigner du Sud, de Rome et la Méditerranée; le salut était dans la culture classique"16.

C'est peu être la première fois que le jeune architecte entre en contact avec l'exaltation de la culture classique, et intègre les motivations de l'auteur, au point d'en commenter les raisons avec enthousiasme, expliquant aux marges du même texte: "... entièrement d'accord avec l'esprit général et génial (...) En ce qui me concerne, ce livre vient aider favorablement mon orientation. Il provoque, l'examen, les déductions normales, claires, lumineuses; relâche la poignée allemande. Dans un an, à Rome, je le relirai, et avec des croquis je fonderai ma discipline du Jura, de Neuchâtel"177. Durant cette phase se précise la volonté d'étudier personnellement les architectures romaines et cela se confirme dans sa correspondance, du 2 décembre de la même année avec la famille ${ }^{18}$ et dans une lettre expédiée un peu plus tard à L'Eplattenier. "...c'est la mon révolution (...) j'ai aussi un livre splendide de dorique, de ionien, de corinthien, de cet art romain fait de voutes colossales et de grands murs pleins. Et, depuis des mois, mes idées se fixent. Je n'irai point en Amérique, ni en Flandres. Mais je resterai ici jusqu'à ce que je sache la langue, abrégeant peut-être mon séjour chez. Behrens pour aller à Dresde pour bâtir chez Tessenow l'Institut Jacques Dalcroze, suivant la proposition qui m'à été faite. Puis je ferai mon voyage d'études en Allemagne et ensuite irai me recueillir. Ou? À Rome"19.

Dans une lettre successive du 10 janvier 1911 à L'Eplattenier, Jeanneret faisait référence à deux volumes inhérents à l'architecture romaine: "J'ai, ici, un splendide livre sur cet art romain, fait de voutes colossales e de grands murs pleins (...) Puis je ferai mon voyage d'études en Allemagne et ensuite irai me réfugier. Où? Á

\footnotetext{
${ }^{12}$ Talamona, M., “L'ai décidé de finir mes études...sans le rêve. J'avais pour cela songé à Rome”, p. 117, p. 134 n. 18. dans Talamona, M., 2012, op.cit.

${ }^{13}$ Dumont, M.-J., Dall' Art Nouveau all'avanguardia, tasselli di una formazione, p. 29 dans Talamona, M., 2012, op.cit.

${ }^{14}$ Turner, P. V., La formazione di Le Corbusier, idealismo e Movimento Moderno, Milano: Editoriale Jaca Book SpA, 2001, pp. 59, 112-118

${ }^{15}$ Talamona, M., "L'ai décidé de finir mes études...sans le rêve. J'avais pour cela songé à Rome”, p.112 dans Talamona, M., 2012, op.cit.

${ }^{16}$ Criniglia-Vaneyre, A., Les Esentretiens de la villa di Rouet, Genève, 1908, p. 9, dans Turner, P. V., La formazione di Le Corbusier, idealismo e Movimento Moderno, Milano: Editoriale Jaca Book SpA, 2001, p. 113

${ }^{17}$ Criniglia-Vaneyre, A., Les Esentretiens de la villa di Rouet, Genève, 1908, p. 69 dans Turner, P. V., 2001, p.113 op.cit.

${ }^{18}$ Baudouï, R., Dercelles A., (éditer par) Le Corbiusier correspondances. Lettres à la famille 1900-1925 vol I fondation Le Corbusier / Paris-Gollion: infolio Editions, 2011 p. 335 dans Talamona, M., 2012, p.115 op.cit.

${ }^{19}$ Jeanneret, C.-E., lettre à Charles L'Eplattenier, 16 janvier 1911, dans Dumont, M.-J., Lettres a Charles L'Eplattenier, Paris: Editons du Linteau, 2006, p. 258-260 dans Talamona, M., 2012, p.115 op.cit.
} 
Rome. Alors, si vous me voudrez encore... Ah, cher maître, la constatation de mon esthétique aboutie est la seule chose qui me permet encore de faire cette vie" ${ }^{\prime 20}$.

Giuliano Gresleri a identifié dans les deux volumes les œuvres de Pierre Gusman: "Chargé de la Mission archéologique en Italie par le Ministre de L'instruction publique et des Beaux-Arts" ${ }^{\prime 21}$ contenues, selon l'érudit, dans la bibliothèque de l'école de Le Chaux-de-Fonds. Les textes sont dédiés à Pompéi et à Villa Adriana. Durant la description des volumes on peut remarquer la présence de représentations des environnements de Pompéi et de la Villa Adriana, "lithographies produites à partir de photos et aquarelles réalisés par l'auteur ou par des élèves de Villa De Médicis qui «perfectionnaient» actualisant les dessins de Pirro Ligorio e Piranesi"22.

Donc, il se pourrait que ce soit la première fois que Jeanneret rencontre Piranesi, même si indirectement. Les représentations reportées dans le texte et transmises par les gravures devaient probablement conserver et transmettre les caractéristiques physiques des objets en question, tout comme les encadrements en perspective et, malgré l'inévitable synthèse, les traits essentiels du langage piranésien.

Un autre élément qui contribue à stimuler son intérêt pour l'architecture romaine, vient toujours de Berlin; durant la visite au Unterrichtsanstalt des Königlichen Kunstgewerbemuseum (1911) Jeanneret a la possibilité d'observer certaines gravures de Piranesi et note une déclaration qui documente son grand intérêt: "tout ce ci gravures de Piranesi admirable. Mais voir à Rome la collection complète et choisir les meilleurs" ${ }^{\prime 23}$. Son attention a été captivé par les architectures exposées, mais peut être aussi par la capacité expressive des œuvres piranésiennes.

Peu après cet événement le jeune Jeanneret entreprend son Voyage d'Orient qui le conduit à travers de nombreuses villes pour rejoindre enfin Rome le 14 Octobre 1911.

C'est une occasion pour se dédier à la découverte des œuvres architecturales et artistiques majeures de la ville, qui est envahie par de nombreuses manifestations pour la célébration du cinquantenaire de l'Unité d'Italie.

Parmi les évènements artistiques auxquels adhère Jeanneret on rappelle la série d'expositions rétrospectives à Castel Sant'Angelo, où se trouve l'exposition topographique romaine, qui contient une ample collection de vues de Rome. Parmi les œuvres on trouve des peintures de Canaletto, Van Wittel, les œuvres de Vasi, Pannini et Piranesi $^{24}$ et les aquarelles de la Roma Sparita de Ettore Roesler Frantz ${ }^{25}$, dont Jeanneret acquiert certaines reproductions; une série d'œuvres qui pour la plupart présentaient les vues d'une Rome qui suintait de paysages mélancoliques, représentant l'aspect dévasté de la ville Eternelle.

\section{Les ruines dans le paysage romain}

Durant le séjour romain de nombreuses architectures attirent l'attention de Jeanneret, qui passe ces journées à noter sur ses cahiers croquis et impressions sur les architectures qui l'intéressent plus. L'ensemble des données

\footnotetext{
${ }^{20}$ Jeanneret, C.-E., lettera a Charles L'Eplattenier, 10 Janvier 1911, AFLC dans Gresleri, G., Dalla Villa alle Ville: Jeanneret e Adriano, dans Talamona, M., 2012, p. 138 op.cit.

${ }^{21}$ Gresleri, G., Dalla Villa alle Ville: Jeanneret e Adriano, in L'Italia di Le Corbusier, dans Talamona, M., 2012., p. 138 op.cit.

${ }^{22} i d .$, p. 138

${ }^{23}$ Jeanneret, C.-E., Voyage d'Orient. Carnets, Milano: Electa, 1987, p. 17 dans Talamona, M., "L'ai decidé de finir mes études...sans le rêve. J'avais pour cela sogné à Rome”, dans Talamona, M., 2012, p. 117 op.cit.

${ }^{24}$ Guida generale delle Mostre Retrospettive di Castel Sant 'Angelo, Istituto Italiano di Arti Grafiche, Bergamo 1911 dans Talamona, M., 2012, p. 117 op.cit.

${ }^{25}$ Talamona, M., "L'ai décidé de finir mes études...sans le rêve, p.124 J'avais pour cela songé à Rome”, p. 124 dans Talamona, M., 2012, p. 117 op.cit.
} 
récoltées dans le $4^{\circ}$ et $5^{\circ}$ Carnet - dédiés aux expériences architecturales - et les correspondances échangées durant cette période communiquent une alternance d'émotions opposées provenant de l'expérience du paysage romain. Dans une des lettres adressées à Auguste Klipstein, en décrivant le bilan des lieux visités durant le Grand Tour il affirme : "Rome n'a pas de silhouette, pas d'âme. Oh Istanbul! Oh Athènes! Mais Rome a les vieux Romaines de la brique cuite, et le bon Dieu a permis que tous les revêtements de marbre aient été volés. Alors $c^{\prime}$ est magnifique, unique, subjuguant. Ça, c'est du musée pour architecte" ${ }^{\prime 26}$.

Au rejet dédaigneux de l'image urbaine correspond la révélation de la ruine, exaltée en vertu du pouvoir qu'elle a d'extrapoler ceux qui sont, selon le jeune, les contenus le plus authentiques et significatifs de l'architecture romaine. Les ruines révèlent en effet les masses dénudées, épurées des revêtements décoratifs. Ces déclarations semblent faire écho, à distance de cinquante ans, à certaines réflexions laissées par Viollet-le-Duc: “...Les ruines des monuments vraiment romains, c'est-à-dire édifiés suivant la donnée romaine, ont du style. Restaurez la plupart de ces ruines, remettez en place les ordres, les encadrements, les bandeaux, les ornements enlevés par les barbares ou par le temps, vous verrez le style propre à ces grandioses constructions s'effacer, à mesure que vous y replacerez ces applications empruntées à un autre art, à un autre ordre d'idées, à un autre principe de structure (...), ${ }^{, 27}$.

Ces concepts refont surface lors de l'étude de certaines architectures mieux conservées, comme le Colisée, durant laquelle Jeanneret copie certaines vues du Piranesi et affirme: "pourrait servir de base à une discussion sur l'unité monumentale où il n'y a qu'utilité ou (il n'y a) point d'ordres d'architecture mais seulement une belle expressivité architecturale ${ }^{, 28}$. Encore une fois les ruines en tant que telles sont objet de réflexions utiles à comprendre la valeur de la masse isolée, bien exprimée dans les vues évocatrices de Piranesi. Ces amas émergent dans le paysage urbain et deviennent une autre occasion pour une réflexion sur le paysage - "un paysage urbain à composé, 29 - contribuant à la formation des principes contenus dans le texte Vers une architecture.

La fascination de Le Corbusier pour le paysage archéologique romain trouve, dans l'interprétation qu'en donne Piranesi, un intérêt renouvelé vers la forme phénomènique telle qu'elle se présente. L'attitude déjà manifestée par l'architecte envers le dessin des ruines, à coté des dessin des précises reconstitutions historiques, souligne cette attention; Piranesi renforce la nature des ruines, et il rende clairement lisible les éléments qui avaient déjà fasciné Le Corbusier: ces amas ruinés laissent apprécier la pure venustas de l'architecture, dénudés par le temps de la firmitas et de la utilitas.

"Face aux antiquités Romaines ou Grecques ou de la Renaissance, (l'architect) arrive à s'isoler de chaque stéréotype de langage pour saisir à l'état pur les émotions suscitées par une séquence d'espaces, par un particulier effet de clair-obscur, par le jeu des volumes, par la texture des matériaux, par les relations avec le paysage, par les signaux par lesquels les formes témoignent les présences des hommes ou stimulent l'imagination de l'observer à les penser" ${ }^{\prime 30}$.

\footnotetext{
${ }^{26}$ Jeanneret, C.-E., lettera a August klipstein, 28 Ottobre 1911, BV LC/102/1267 dans Talamona, M., L'ai decidé de finir mes études...sans le rêve. J'avais pour cela sogné à Rome, p. 131, dans Talamona, M., 2012, op.cit.

${ }^{27}$ Viollet-le-Duc, E.-E., Dictionaire raisonné dell'architecture française di XIe au XVIe siècle, Paris, 1866, Tom. $8^{\circ}$, ad vocem "Style"

${ }^{28}$ Jeanneret, C.-E., doc. FLC B2 (20) 245, dans Cohen, J.-L., Roma come lezione, dans Talamona, M., 2012 p. 188 op.cit.

${ }^{29}$ Jeanneret, C.-E., Carnet 4, Milano: Electa, 1987, p. 140, Le Corbusier, Vers une architecture, Parigi, 1923, p. 128 et Talamona, M., 2012, p. 131 op.cit.

${ }^{30}$ Tedeschi, E. G., Le lezioni di Tivoli, dans Mauri, A. (sous la direction), Le Corbusier a Villa Adriana, un atlante, Bologna: Alinea editrice s.r.l., 1999 p.10
} 
Le paysage archéologique de la Villa Adriana se présente, ainsi, dans sa forme brut, comme matière de projet et référence spatial, comme "ensemble monumental, très structuré, dans lequel s'intègrent sans rupture de continuité épisodes sans cesse variés, qui offrent surprises en boucle, organisations spatiaux de énorme effet, grâce à une régie très savante, dont les résultats surclassent souvent la qualité architecturale spécifique des éléments particuliers ${ }^{\prime \prime 31}$.

La vision de l'archéologie donne à Le Corbusier une leçon qui complète la mathématique perfection de l'antique, et plonge, au contraire, dans l'imperfection des fragments et l'effritement perceptif des rigoureuses matrices du projet. L'espace inspiré à l'espace archéologique est un espace absolu, détaché de son lien avec le plein, un "corps atmosphérique immatériel traversé par corps architecturaux de mesure inférieure lui subordonnés" 32.

Son héritage, que Franco Purini ressente dans la "antiquité virtuelle ${ }^{, 33}$ de la ville des Lumières, caractérisé par la fragmentation d'une unité perdue, par la stratification des bâtiments construits et reconstruits, par l'effritement dimensionnel d'un forma urbis perdue, se retrouve dans une "échelle urbaine réduite à peu environnements résiduelles, hétérogènes et contradictoires" ${ }^{34}$ et dans une "espace complexe, au même temps continu et discontinu (...) indéchiffrable, trop variés dans ses composantes pour être considéré comme quelque chose doté encore de spécifiques matrices génératrices ${ }^{\$ 3}$.

Les considérations sur la ville reflètent les composition du Maître vénitien, que recherche sa réalité virtuel sur la base de la structure logique de le espace archéologique. Les pièces sont des éléments isolés, combinés "niant le connexions syntaxiques entre les parties en faveur des libres associations thématiques "36 selon une procédure paratactique, innée dans la réalité visible du paysage archéologique, et exalté dans les œuvres de Piranesi.

"Dans la Villa Adriana plans horizontaux établis en entente avec la plaine romaine; montagnes qui ferment la composition établie, d'ailleurs, par rapport à eux ",37: la réflexion de Le Corbusier "n'est pas, naturellement, un élément objectif, inhérent à la façon de bâtir romaine connaissable par une analyse philologique des ruines; elle est, plutôt, une interprétation ciblée, la découverte d'un artiste-architect auquel n'intéresse pas la reconstruction philologique, mais le sens que la culture moderne peut donner au héritage de l'histoire, ${ }^{\text {,38. }}$.

\section{De la Villa Adriana à la Haute Cour de Justice à Chandigarh}

Parmi les plus importantes annotations sur le séjour romain duré onze jours (du 14 au 28 octobre, 1911) ) $^{39}$ l'intérêt est captivé par la visite de Villa Adriana à Tivoli et certaines réflexions sur Villa d'Este. Le jeune Jeanneret dédie trois jours à l'étude de l'architecture et des ruines, qui se concluent avec cinquante-neuf pages de dessins et notes, contenues en partie dans le Carnet 4 et dans le Carnet 5 tout entier.

Les réflexions sur l'ensemble des antiquités de Villa Adriana sont nombreuses, tout comme les ambitions qu'il transporte dans sa successive production architecturale et théorique. Celles-ci seront présentées en premier plan

\footnotetext{
${ }^{31} i d .$, p. 10

${ }^{32}$ Purini, F., Attualità di Giovanni Battista Piranesi, Melfi:Libria, 2008, p. 23

${ }^{33}$ id., p. 9

${ }^{34} i d .$, p. 11

${ }^{35} i d .$, p. 11

${ }^{36} i d .$, p. 12

${ }^{37}$ Le Corbusier, Verso una Architettura, Cerri P.; Nicolin P. (sous la direction) Milano: Longhesi, 5 ed., 2003, p. 159

${ }^{38}$ Denti, G., La ricerca delle leggi e dell'ordine, dans Mauri, A., 1999, p. 13 op. cit.

${ }^{39}$ Petit, J., Le Corbusier. Lui-même, Genève: Edition Rousseau, 1970, pp. 24-44
} 
durant la publication du projet de Sainte-Baume dans l'Euvre Complète ${ }^{40}$ et dans d'autres réalisations telles que Ronchamp et Chandigarh" ${ }^{41}$ En outre "la Villa Adriana figure, même si pas expressément mentionnée, dans des croquis illustratifs de Urbanisme: l'intérêt n'est pas donné par leur relation avec le contexte, mais en tant que signe de la présence de ce monument dans les souvenirs de Le Corbusier, comme preuve de l'importante impression qui il a suscité à l'auteur, ${ }^{, 42}$.

Les nombreux croquis effectués durant le séjour clarifient l'attente de la visite: le jeune produit de nombreux dessins de ruines, parcourant les diverses parties du complexe, et évoque les encadrements en perspectives présents dans les images de Pierre Gusman, dans lesquels l'auteur retravaille les gravures de Pirro Ligorio et Piranesi. Ces éléments sont utiles à Jeanneret pour fixer dans la mémoire le souvenir des masses romaines, comme dans la représentation du mur du Pecile, où est utilisé le même encadrement en perspective qui de manière synthétique met en lumière les géométries originales du complexe et leurs rapports avec le paysage naturel ${ }^{43}$ (image 1$)$.

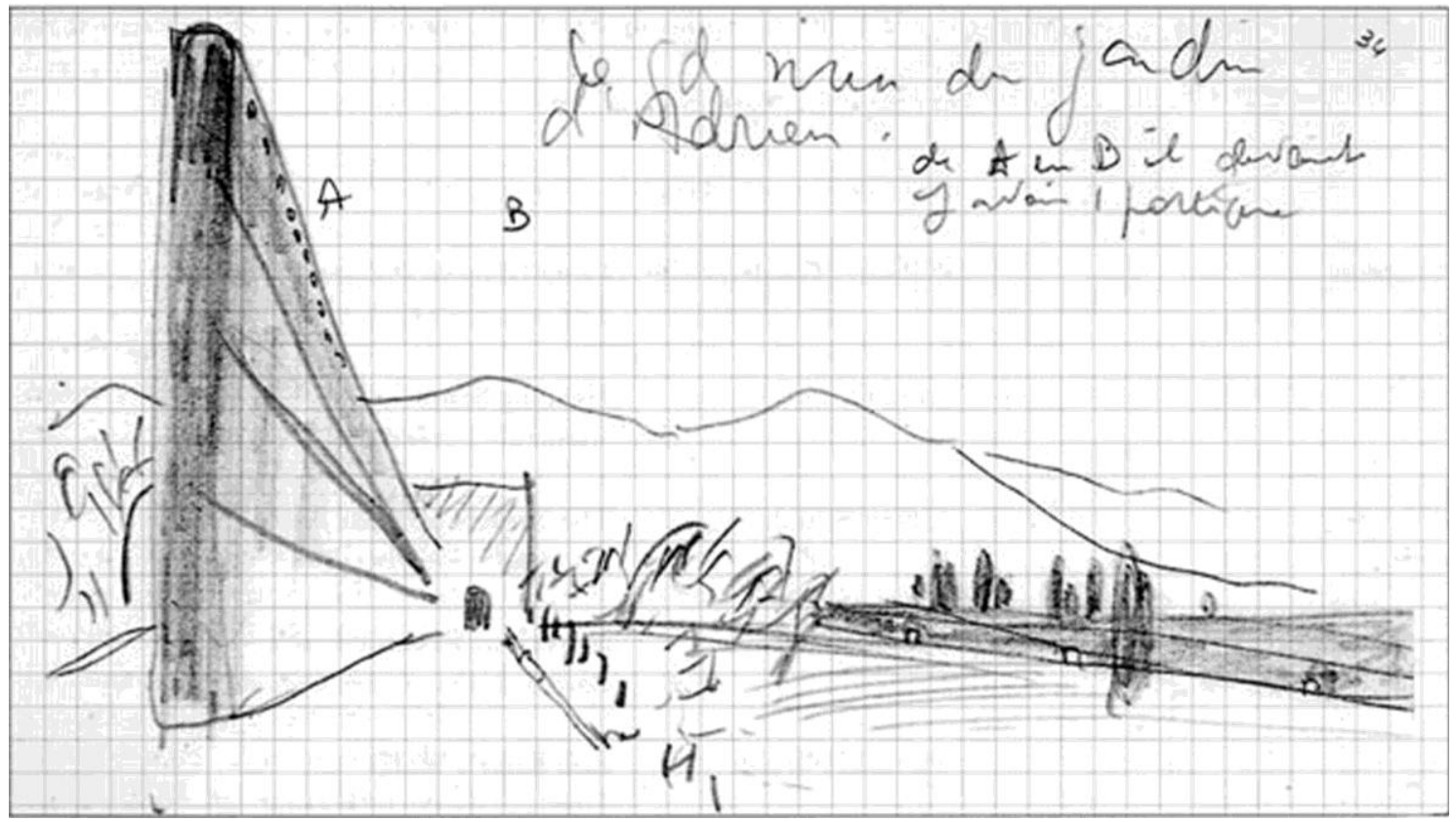

1. Jeanneret, C.-E., Carnet 5, p. 34: Esquisse de Villa Adriana, 1911.

Le deuxième croquis (image 2) représente le Praetorium ${ }^{44}$ - logements des Gladiateurs - avec un dessin précis des géométries originales de la structure, dépouillé de l'étage supérieur originel dans lequel exaltent les gigantesques arches; dans le dessin suivant ${ }^{45}$ (Carnet 5, p. 79) il rappelle ce qu'il reste de l'antiquité, en soulignant le caractère de la ruine. Une représentation qui suggère la volonté d'emprisonner la mémoire de la ruine en tant que telle, où la fragmentation de l'étage supérieur et la présence de la nature captivent son intérêt tout autant que les géométries compositrices précédentes. Une confirmation supplémentaire qui pourrait étayer

\footnotetext{
${ }^{40}$ Le Corbusier, Oeuvre Complète 1957-1965 Vol. VII, Zurich: Les editions d'architecture, 1967 et Gresleri, G., Dalla Villa alle Ville: Jeanneret e Adriano, dans Talamona M., 2012, p. 149 op.cit.

${ }^{41}$ Gresleri, G., Dalla Villa alle Ville: Jeanneret e Adriano, dans Talamona, M., 2012, p. 149 op.cit.

${ }^{42}$ Tedeschi, E. G., Le lezioni di Tivoli, dans Mauri, A., 1999, p.11 op. cit.

${ }^{43}$ Gresleri, G., Dalla Villa alle Ville: Jeanneret e Adriano, dans Talamona, M., 2012,. pp. 140-141 op.cit

${ }^{44}$ Jeanneret C.-E., Carnet 5, Milano: Electa, 1987, p. 78, dans Gresleri G., Dalla Villa alle Ville: Jeanneret e Adriano, dans M. Talamona, 2012, p. 146 op.cit.

${ }^{45}$ id., p. 79
} 
l'hypothèse d'une reprise d'une vue piranésienne, Rovine di uno degli alloggiamenti dé Soldati presso ad una delle eminenti fabbriche di Adriano nella sua Villa di Tivoli ${ }^{46}$ (image 3), que Jeanneret a vue indirectement dans les textes de Pierre Gusman ou durant la série d'expositions rétrospectives à Castel Sant' Angelo, visitées depuis peu.

La représentation du croquis du jeune et l'image de Piranesi contiennent la volonté de maintenir le caractère actuel de l'antiquité. On pourrait supposer que Jeanneret se soit à nouveau penché, en direct, sur l'architecture qu'il avait déjà vue précédemment dessinée, et dont il assimile la connaissance des constructions et aussi la capacité évocatrice des ruines.

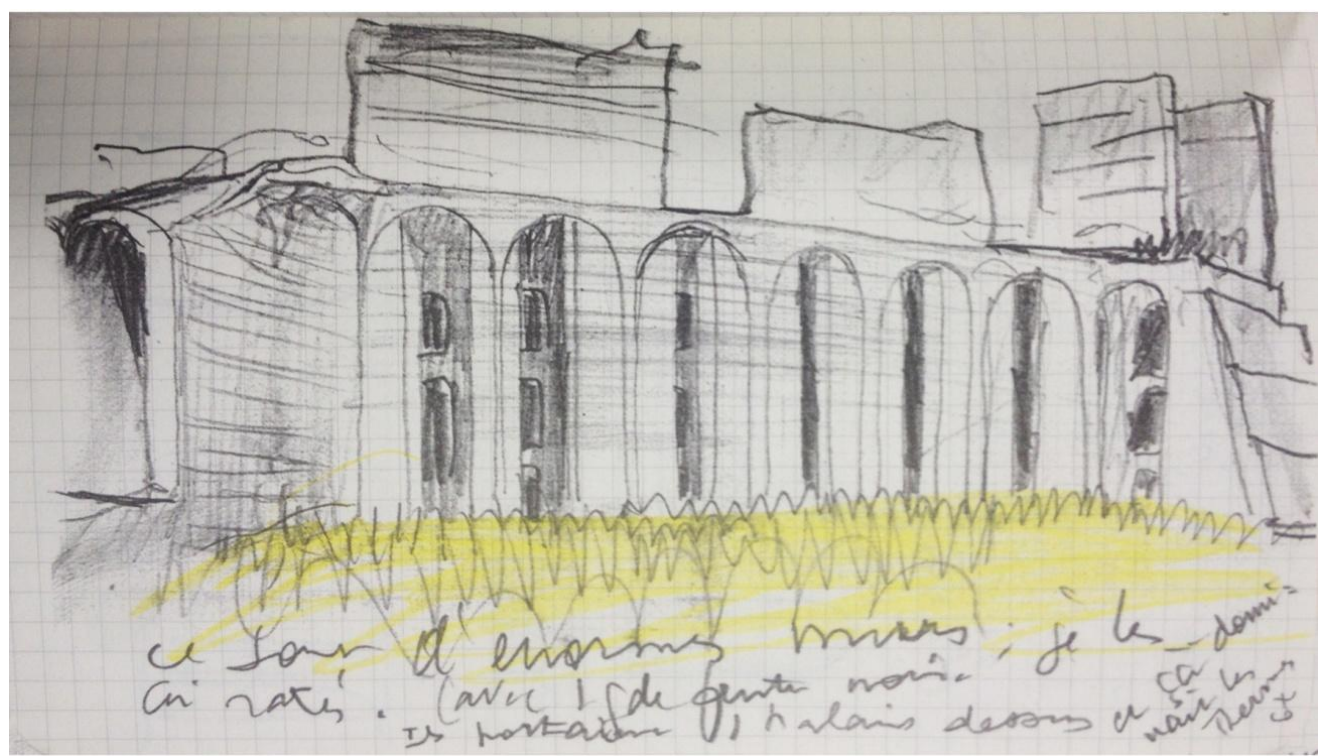

2. Jeanneret, C.-E., Carnet 5, p. 79: Esquisse de Villa Adriana, Le Praetorium, 1911.

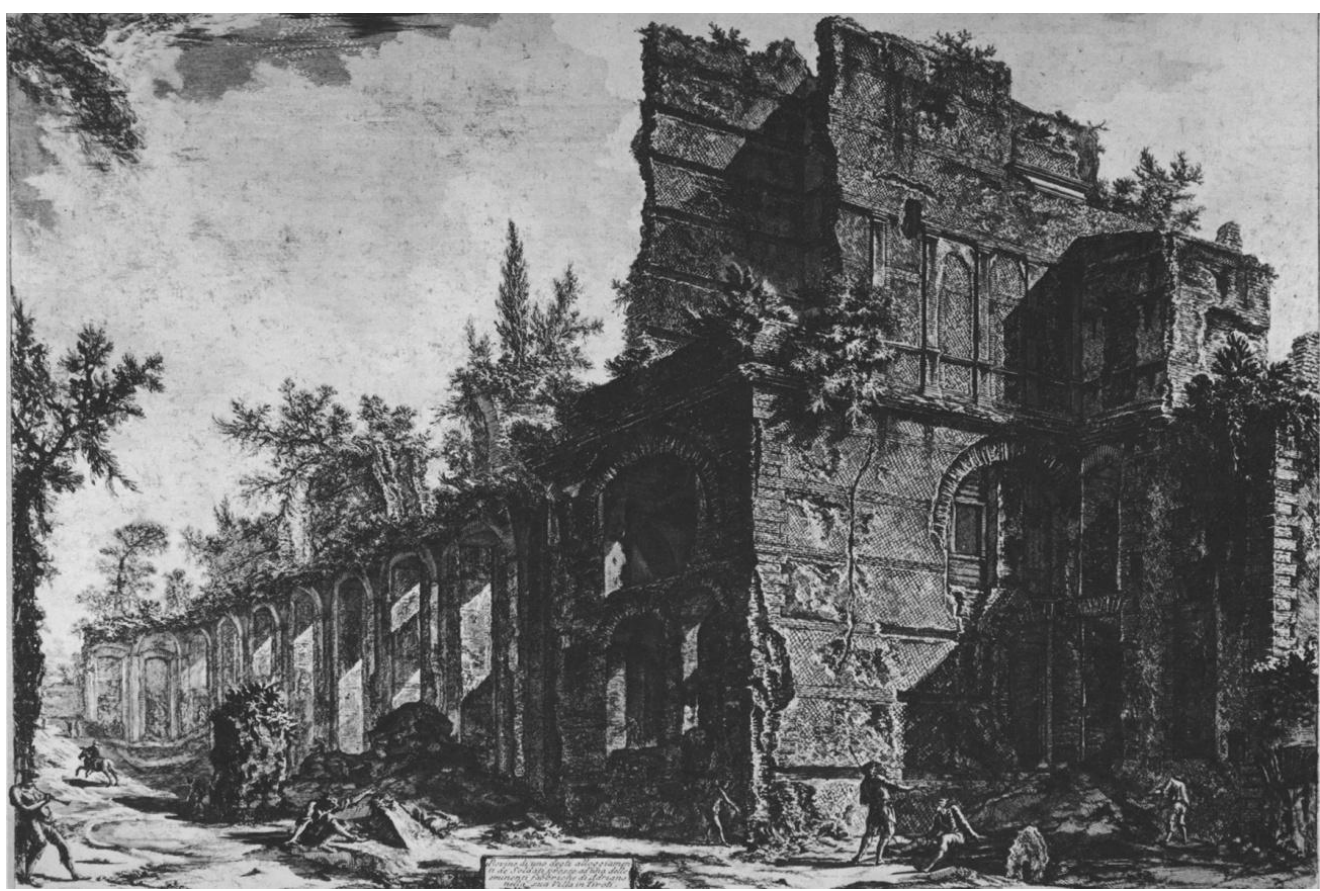

3. G. B. Piranesi, Rovine di uno degli alloggiamenti dé Soldati presso ad una delle eminenti fabbriche di Adriano nella sua Villa di Tivoli.

${ }^{46}$ Conti V.; Piranesi F., Piranesi, vedute e antichità di Tivoli, Roma: Centro Stampe de Vittoria, 1996, Tav. 22 
Le croquis du Praetorium resurgit, inspirant la composition de Ronchamp et les grandes arches qui composent l'édifice de la Haute Cour à Chandigarh ${ }^{47}$. "Si le souvenir de Villa Adriana est rappelé ainsi, soudainement, ce n'es pas seulement parce que Le Corbusier est allé à Tivoli et a regardé longtemps ce lieu si suggestif, mais, surtout, parce que il choisit de dessiner ce qui assume effectivement le rôle de souvenir pour l'Architect" ${ }^{\prime \prime 8}$. L'architect, donc, retrouve dans ses souvenirs "un microcosme qui réunit types de bâtiment et caractères spatiaux significatifs de la façon de construire romaine: les techniques des murs en briques et des voutes en béton, l'articulation et l'ouverture des enveloppes qui établissent les rapports entre intérieur et extérieur, la captation de la lumière qui valorise l'espace interne et donne un significat suggestif aux espaces sous-sol",49 (image 4).

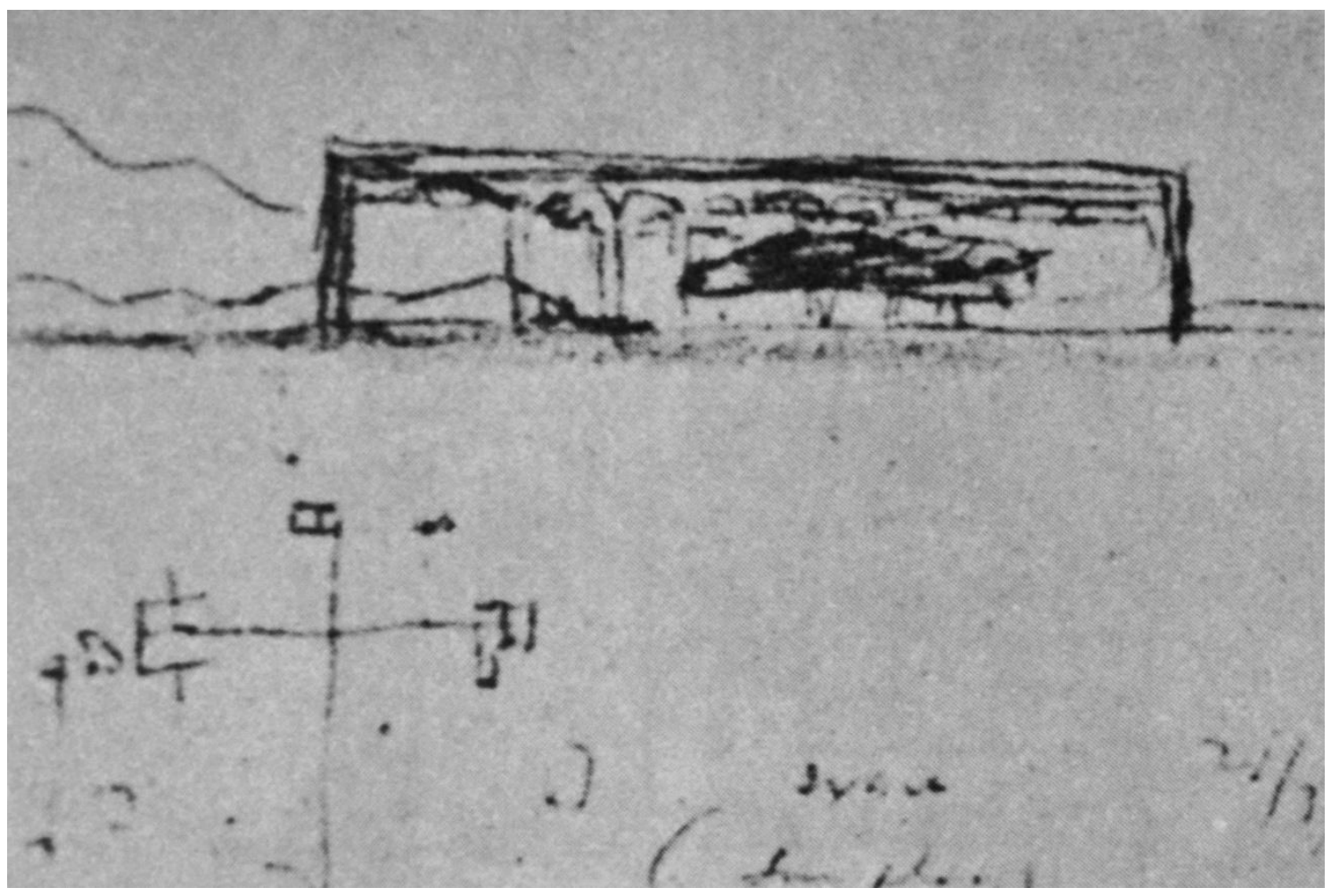

4. Le Corbusier, Premier croquis conceptuel de la Haute Cour de Chandigarh.

La géométrie de la forme de la façade de la Haute Cour (image 5), définie par le subtil parallélépipède qui embrasse et ferme l'édifice grâce à un signe pur, bloque le système continu des puissantes arches romaines. Ce signe précis encadre la structure et crée une friction avec le vide intérieur, où l'on perçoit la fluidité spatiale des voutes.

L'image du Praetorium gravée par Piranesi exalte la légèreté de la construction, accentuant les faisceaux lumineux qui traversent la structure; un mécanisme qui laisse pressentir une spatialité complexe, au-delà de la première couche de pierre du Praetorium.

\footnotetext{
${ }^{47}$ Gresleri, G., Dalla Villa alle Ville: Jeanneret e Adriano, dans M. Talamona, 2012, p. 136-150 op.cit.

${ }^{48}$ Danièle P., la cappella di Rochamp, come esempio del processo creativo di Le Corbusier, dans: AA.VV. Le corbusier 18871965, Mialno: Electa, 1993, p. 168

${ }^{49}$ Denti, G., La ricerca delle leggi e dell'ordine, dans Mauri, A., 1999, p. 12 op. cit.
} 


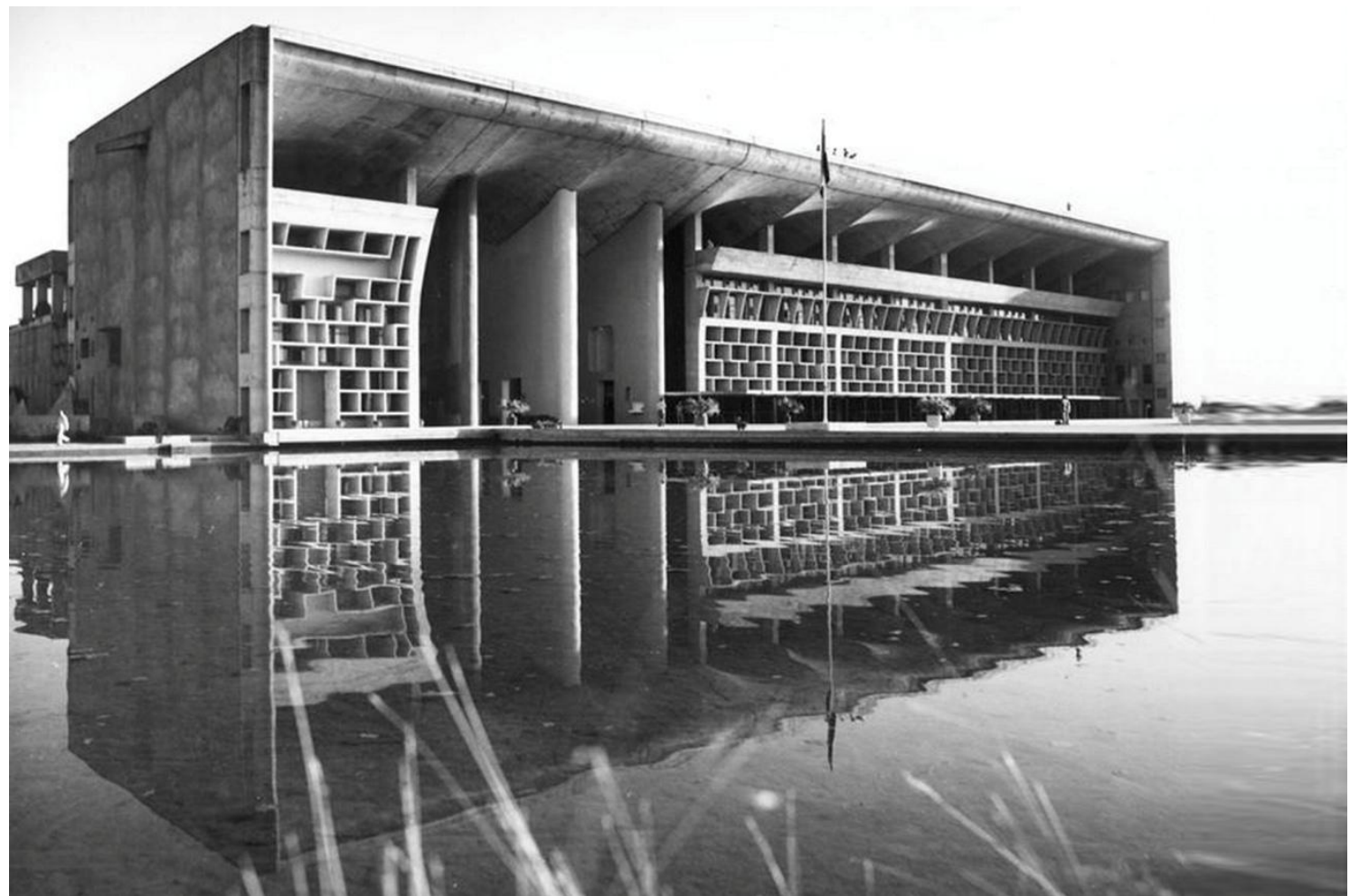

5. Le Corbusier, Haute Cour de Chandigarh, 1952.

Cet espace interne vit des éléments menus, apposés au grand édifice, qui d'un coté suggèrent la fragmentation et l'effritement de l'ensemble, caractéristique primaire des ruines, et d'un autre avivent le gigantisme de la figure qui frise l'infini vers l'horizon. De la même façon, l'Haute Cour naît comme un ordre géant d'arcades creuses qui laissent regarder au-delà, et qui révèlent les organismes fonctionnels greffés sur leur structure, générant une relation osmotique avec l'extérieur. Le système du vide qui traverse l'espace interne et externe, particularité de toutes les architectures en ruine, est accentué dans l'Haute Cour: le bâtiment manque de façade, d'un dispositif architectonique de construction du front, mais au contraire caractérise son aspect extérieur par la visibilité de sa nature intérieure. Le système de vide laisse traverser ses éléments par le soleil, tout comme à Villa Adriana "la lumière modèle les formes de la nature et l'architecture, et leur relation mutuelle intéresse Le Corbusier, qui la souligne dans ses croquis ${ }^{, 50}$ (image 6).

Grâce à l'espace ouvert sous-jacent les arcades, le bâtiment de l'Haute Cour cherche une relation avec le milieu extérieur et son paysage bâti, un lien émotif avec les parties d'un ensemble dilaté et hétérogène, selon une volonté déjà invoquée lors de la conception du projet de l'ensemble du Capitole, quand, en 1951, "l'architecte signe la grande prospective où l'on voit, sous le grand cadre parasol de la Haute Cour, une vue piranésienne sur laquelle se détachent les bâtiments" "51 (image 7), manifestant l'intention de construire un système composé par éléments décousus, paratactiquement approchés, libres dans l'espace atmosphérique décrit de l'Himalaya à l'arrière-plan. Une liste de vibrations qui se détachent dans l'espace indicible de l'écoute.

\footnotetext{
${ }^{50} i d .$, p. 12

${ }^{51}$ Papillault, R., Chandigarh et Le Corbusier, Creation d'une ville en Inde, 1950-1965, Toulouse: Edition Poesis, 2011, p.234
} 


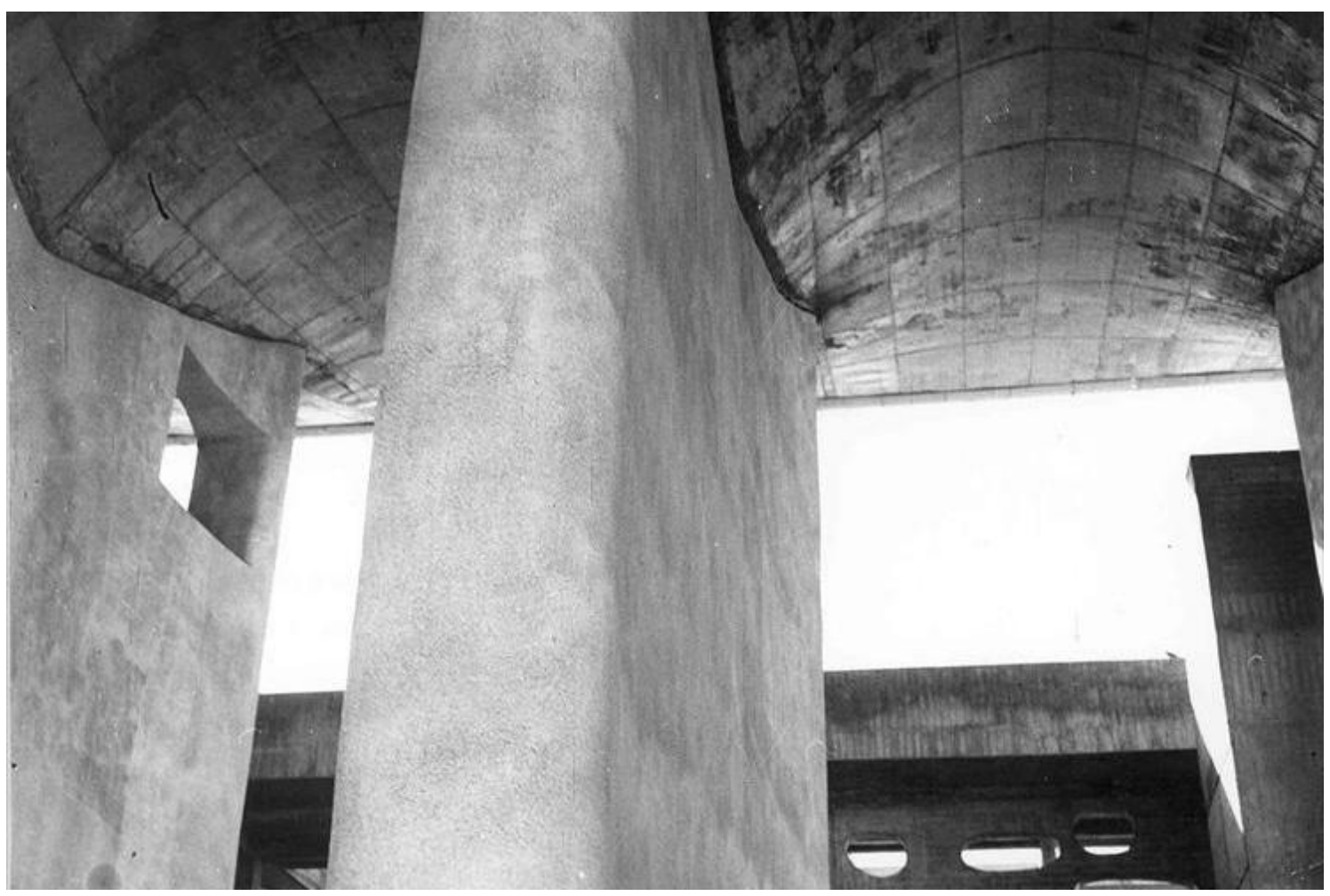

6. Le Corbusier, Haute Cour de Chandigarh, 1952.

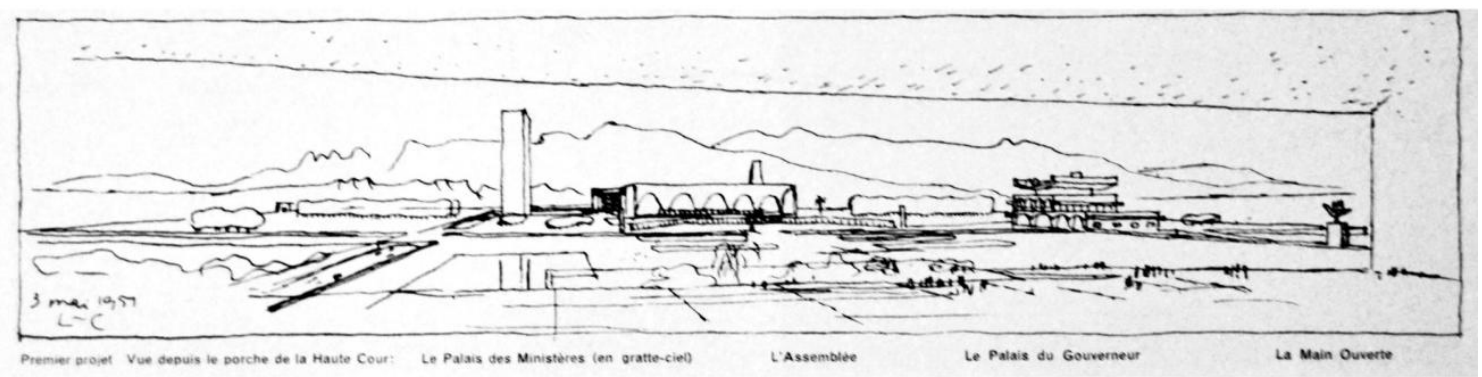

7. Le Corbusier, Perspective sur le Capitole signé Le Corbusier (le Sécretariat est encore une tour, l'Assemblée présente une élévation à arcades, le Palais du Gouverneur et la Main Ouverte ont leur forme presque définitive), 3 mai 1951.

Le Corbusier lie le bâtiment au sol, le dépouillant du socle supposé au début ${ }^{52}$, de façon que les douze piliers qui définissent le rythme des treize travées paraissent enracinés à terre. L'accentuation de l'espace module l'insertion des volumes fonctionnels, coincés entre les butées et renfermés en haut par un évident plancher, dessinés par un système de façade constitué d'une grille irrégulière de ciment qui crée un "lien visuel entre l'observateur et

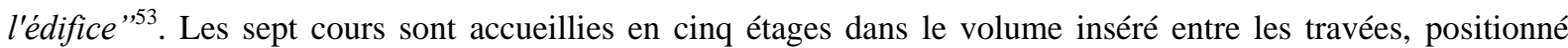
asymétriquement. Une asymétrie qui travail avec les corps fonctionnels à la base de l'édifice, éléments indépendants par rapport au système vouté qui semblent suggérer la présence d'éléments moyenâgeux superflus, qui seraient des systèmes superposés à la pure géométrie recherchée dans l'architecture romaine. Ceux-ci déséquilibrent la structure principale, en définissant une superfétation majeure, et déterminent une arythmie des travées coïncidente avec l'entrée. De l'autre coté, marqué par une seule travée plus grande, l'autre plein de la

\footnotetext{
${ }^{52} i d$.

${ }^{53}$ Serenyi, P., Senza tempo ma del suo tempo: l'architettura di Le Corbusier in India, dans Brooks, H. A., Le Corbusier, Milano: Electa, 2001, p. 202.
} 
composition se place près le vide de l'entrée, manifestant une rupture entre les parties. Le vide du hall, souligné par les travées latérales plus étroites, est l'occasion pour créer un espace à toute hauteur, vertigineux, où sont visibles les "chainettes ${ }^{\prime 54}$ des arcades, en souvenir des substructions romaines. Dans cet espace creux s'inscrit un mur à l'arrière-plan, qui rend la majestueuse entrée semi-ouverte. Le mur au fond, plein linéaire percé par des ouvertures difformes, est structure de la rampe, et crée une complexité spatiale interne peu prévisible de loin, dramatisée par le soleil filtrant. La rampe serpente entre les galeries à différentes étages qui distribuent les fonctions, posés a l'arrière et enveloppés par un épais brise-soleil qui concourent au dessin di front postérieur, sur lequel s'imprime le volume de la salle des pas perdus.

De l'autre côté, le brise-soleil qui module le front antérieur, orienté vers les autres édifices du Capitole, se recourbe pour chercher un dialogue, pour suggérer ainsi une tension, grâce à une déformation qui reste toujours ouverte, qui ne se referme pas dans la géométrie du bâtiment. "Les trois grands objets désireux cherchent de briser leur solitude (...) L'Haute Cour à travers l'inflexion des brise-soleil" ${ }^{p 5}$ essaye de évoquer une unité perdue, de faire allusion aux autres éléments qui constituent l'espace du Capitole, comme les reins d'un arc effondré renvoient à leurs complément (image 8).

C'est précisément en ce système d'émergences solitaires que s'accomplit la suggestion du paysage archéologique, l'inachèvement propre des fragments, produits d'une matrice difficilement intelligible, où "rien n'aide l'œil à se situer par rapport à ces trois personnages, (...) pas d'allusions prospectives, pas de triangulations formelles, qui établissent une conversation dont l'oreille humaine n'est adéquate à saisir que faibles et déformés échos ${ }^{\prime \prime 56}$.

La matrice géométrique du projet laisse de la marge à la correction sensible, à l'émergence de la sensibilité de l'architecte à travers des mailles de la structure logique dont lui même s'était équipé. L'effort initial pour le Capitole, sur lequel il concentra les attentions majeurs, en laissant aux lignes tracées par Mayer l'organisation du plan urbain, convergea en une composition orientée sur grands axes et carrées pour structurer le projet de l'espace ouvert et les éléments le composant, suivant le système métrique-proportionnel du Modulor.

Dans la recherche de tracer des références spatiales, de reconnaître des pierres angulaires pour projeter la grande esplanade représentative du Capitole indien, Le Corbusier recourt à sa mémoire intérieure, émotionnelle, aux lointains souvenirs de sa jeunesse, pas pour le remaniement de figures et des allusions mécaniques à architectures déjà vue, mais pour rechercher la même charge émotionnelle évoquée par les ruines du paysage $\operatorname{archéologique~}^{57}$, innée dans la composition de la Villa Adriana, dans la magnificence de l'ensemble comme elle est apparue à ses yeux.

\footnotetext{
${ }^{54}$ Papillault, R., 2011,. p. 232 op. cit

${ }^{55}$ Tafuri, M. Macchine e Memoria, dans Brooks, H. A., 2001 p. 255 op. cit.

${ }^{56}$ id., p. 252

${ }^{57}$ Bellini, F., Il conflitto irriducibile tra raziocinio e natura primordiale, dans Casciato, M., Le Corbusier e Chandigarh, ritratto di una città moderna, Roma: Edizioni Kappa, 2003, pp. 107-116
} 


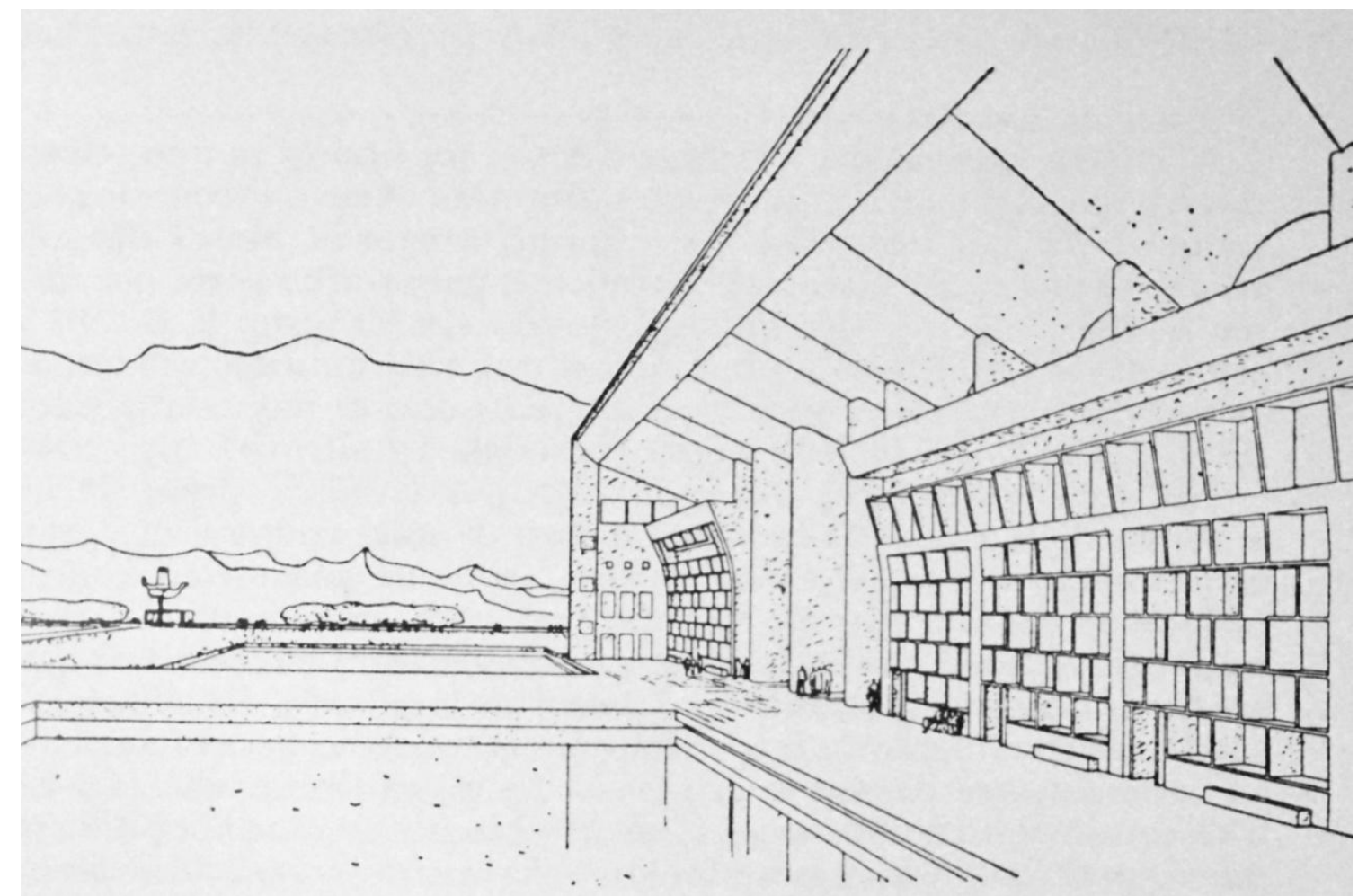

8. Le Corbusier, Perspective de la Haute Cour de Chandigarh.

Il ne s'agit plus seulement de la géométrie proportionnelle, des grandes axes générateurs faisant allusion à un schéma mental mathématicien presque parfaite, qui conduisent la conception du projet: il décide de sous-tendre la rigueur géométrique à une géométrie déclarée qu'en partie. Comme cela arrive dans les architecture fragmentées par le temps, qui expriment les émotions juste grâce à l'absence des pièces, à la perte de la vérité géométrique présente dans leur conception originale, comme les lignes-guide qui structurent la composition du Capitole.

La volonté de construire un paysage architectural qui se base sur des objets isolés, met en évidence sous la lumière l'uniformité de la matière du ciment brut qui devient écho de la matérialité de la ruine, contenue dans les masses romaines laissées à l'abandon ${ }^{58}$; et comme les masses antiques, à Chandigarh les édifices se décomposent sur le plan horizontal, souvenirs de la composition ouverte du complexe de Villa Adriana qui manifeste un "urbanisme organique" et "géographique"59 fait de gigantesques mégastructures linéaires qui suivent un paysage primordial.

\section{Conclusion}

L'intérêt pour les ruines du monde antique, qu'il a approché aussi grâce à l'étude des dessins de Piranesi, a guidé sa sensibilité d'architecte et, en particulier, les expériences faites durant la visite de Villa Adriana ont été pour le jeune Jeanneret un enseignement important, auquel il dédiera en 1922 une précise pensées: "Rome s'occupait de conquérir l'univers et de gérer. (...) Hors de Rome, ayant de l'air, ils ont fait la Villa Adriana. On y médite sur la grandeur romaine. Là, ils ont ordonné. C'est la première grande ordonnance occidentale. Si l'on évoque la

\footnotetext{
${ }^{58}$ Von Moos, S., «L'Europe après la pluie» ou le brutalisme face à l'Histoire, dans J. Sbriglio, Le Corbusier et la question du Brutalisme, Paris: Edition Parenthèses, 2013, pp. 66-87

${ }^{59}$ Colquhoun A., Il significato di Le Corbusier, dans Brooks, H. A., 2001, p. 44 op. cit.
} 
Grèce à cette jauge, on dit: "Le Grec était un sculpteur, rien de plus" mais attention, l'architecture n'est pas que d'ordonnance. L'ordonnance est une des prérogatives fondamentales de l'architecture. Se promener dans la Villa Adriana et se dire que la puissance d'organisation qu'est "Rome" n'a encore rien fait, quel tourment pour un homme qui se sent participer, complice, à ce raté désarmant! $!^{60,}$

Les croquis et les réflexions dédiés par l'architect à l'ensemble archéologique de Tivoli, à côté des analyses des vues de Piranesi, manifestent un intérêt vers l'Antique et vers la tension spatiale du paysage archéologique. L'analyse du projet de Chandigarh, et de l'Haute Cour de Justice notamment, montre des suggestions spatiaux et lumineuses, et des éléments de la composition, qui représentent une modalité par laquelle émerge l'empreinte reçue par les architectures Romaines. Cette orientation se superpose à la recherche d'ordre, harmonie et proportion, qui marque la plupart de sa production architecturale et théorique: la fascination des ruines, pour la réaction poétique qu'ils génèrent, au delà du sens et de la fonction de l'architecture, émerge comme composante plastique et irrationnelle plusieurs fois dans ses projets, pour trouver son achèvement dans les œuvres de la maturité, dont le Capitole de Chandigarh.

\section{Crédits des illustrations}

Image 1. Esquisse de Villa Adriana, dans: Jeanneret, C.-E., Voyage d'Orient. Carnet 5, Milano: Electa, 1987, p. 34

Image 2. Esquisse de Villa Adriana, Le Praetorium, dans: Jeanneret, C.-E., Voyage d'Orient. Carnet 5, Milano: Electa, 1987, p. 79

Image 3. Rovine di uno degli alloggiamenti dé Soldati presso ad una delle eminenti fabbriche di Adriano nella sua Villa di Tivoli, dans: Conti, V.; Piranesi, F., Piranesi vedute e antichità di Tivoli, Roma: 1ed. Centro Stampe de Vittoria, 1996.

Image 4. Premier croquis conceptuel de la Haute Cour de Chandigarh, dans: Papillault, Rémi, Chandigarh et Le Corbusier, Création d'une ville en Inde, 1950-1965, Toulouse: Edition Poesis, 2011, p. 215

Image 5. Photo de l'Haute Cour de Chandigarh, dans: www.fondationlecorbusier.fr.

Image 6. Photo de l'Haute Cour de Chandigarh, dans: www.fondationlecorbusier.fr.

Image 7. Perspective sur le Capitole signé Le Corbusier (le Sécretariat est encore une tour, l'Assemblée présente une élévation à arcades, le Palais du Gouverneur et la Main Ouverte ont leur forme presque définitive), 3 mai 1951, dans: Papillault, Rémi, Chandigarh et Le Corbusier, Création d'une ville en Inde, 1950-1965, Toulouse: Edition Poesis, 2011, p. 234

Image 8. Perspective de la Haute Cour de Chandigarh, dans: Le Corbusier, E. Saurwein (sous la direction), Modulor 2, Milano: Gabriele Marzotta Edizioni s.r.l., 1974

\section{Bibliographie}

Abram, Joseph; Bancon Mardges; Baudouï, Rémi, et al., Enciclopedia Le Corbusier, Milano: Electa S.p.a. Milano, 1988

Baudouï, Rémi; Dercelles, Arnaud, Le Corbusier. correspondance. Lettres à la famille 1900-1925 vol I Fondation Le Corbusier, Paris-Gollion, Infolio Editions, 2011

Brooks, Allen H., Le Corbusier 1887-1965, Milano: 1ed. Electa, 1993

Casciato, Maristella, Le Corbusier e Chandigarh, ritratto di una città moderna, Roma: Edizioni Kappa, 2003

\footnotetext{
${ }^{60}$ Le Corbusier-Saugnier, «Architecture, la leçon de Rome », l'Esprit Nouveau, n14 ${ }^{\circ}$ janvier 1922 dans Dubö̈ P., Croquis de voyage et étude, Paris: La Quinzaine Littéraire, 2009, pp. 124-125
} 
Conti, Vincenzo; Piranesi, Francesco, Piranesi vedute e antichità di Tivoli, Roma: 1ed. Centro Stampe de Vittoria, 1996

Duboÿ, Philippe, Croquis de voyage et étude, Paris: 1ed. La Quinzaine Littéraire, 2009

Gravagnuolo, Benedetto, Le Corbusier e l'Antico. Viaggi nel Mediterraneo, Napoli: 1ed. Electa,1997

Jeanneret, Charles-Édouard, L’Esprit Nouveau, Paris: 1 ed., 1920.

Jeanneret, Charles-Édouard, Voyage d'Orient. Carnets, Milano: 1ed. Electa, 1987

Le Corbusier, Le poème de l'angle droit, Milano: 5rd. ed. Electa, 2007

Le Corbusier, Euvre Complète 1957-1965 Vol. VII, Zurich: 2rd. Ed. Les éditions d'architecture, 1967

Le Corbusier, Vers une architecture, Milano: 11rd.ed. I Marmi, 2013

Papillault, Rémi, Chandigarh et Le Corbusier, Création d'une ville en Inde, 1950-1965, Toulouse: Edition Poesis, 2011

Petit, Jean, Le Corbusier lui-même, Paris: 1ed. qui était Le Corbusier, 1968

Purini, Franco, Attualità di Giovanni Battista Piranesi, Melfi: Libria, 2008

Sbriglio, Jacques, Le Corbusier et la question du Brutalisme, Pairs: 1ed. Edition Parenthèses, 2013

Talamona, Marida, L'Italia di Le Corbusier, Milano: Mondadori Electa S.p.A., 2012

Tedeschi, Eugenio Gentili; Denti, Giovanni, Mauri, Annalisa (sous la direction), Bologna: Alinea editrice s.r.l., 1999

Turner, Paul V., La formazione di Le Corbusier, idealismo e Movimento Moderno, Milano: 1ed. It. Editoriale Jaca Book SpA, 2001

Viollet-le-Duc, Eugène Emmanuel, Dictionnaire raisonné de l'architecture française di XIe au XVIe siècle, Saint Julien: 13rd. Ed. Editions de Sancey, 1978-1979 